\title{
Etude Ethnobotanique des Plantes Utilisées en Médecine Traditionnelle dans le Traitement de l'Hypertension Artérielle chez les Peuples du Département de Divo, (Centre-ouest, Côted'Ivoire)
}

\author{
Wangny Akessé Ackah Stéphane, \\ Ouattara Tiéba Victor, \\ Université Félix Houphouët-Boigny, Laboratoire de Physiologie Végétale, \\ UFR Biosciences, Abidjan, Côte d'Ivoire \\ Abrou N'Gouan Emmanuel Joël, \\ N'guessan Koffi, \\ Université Félix Houphouët-Boigny, Laboratoire de Botanique, \\ UFR Biosciences, Abidjan, Côte d'Ivoire
}

Doi:10.19044/esj.2019.v15n24p384 URL:http://dx.doi.org/10.19044/esj.2019.v15n24p384

\section{Résumé}

Dans le but de connaître les plantes médicinales et leurs usages traditionnels sur l'hypertension artérielle, une étude ethnobotanique a été réalisée dans le département de Divo (région du Centre-Ouest forestier). Les enquêtes ethnobotaniques ont permis de visiter 21 villages et de rencontrer, lors des entretiens semi-structurés, 105 tradithérapeutes, sans distinction d'âge, de sexe et de niveau d'instruction. Cette étude a montré que les populations enquêtées emploient 52 espèces de plantes appartenant à 30 familles botaniques, pour combattre l'hypertension artérielle. Parmi ces familles, les Fabaceae avec 17,31\% des plantes prospectées ont été les plus représentées. Ces taxons, dominés par les arbres (50\%), appartiennent majoritairement à la fois à la région Guinéo-Congolaise et à la région Soudano-Zambézienne (GC-SZ) avec 36,54\% des espèces collectées. Les plantes médicinales identifiées ont servi à la préparation de 154 recettes médicamenteuses dont 151 soit 98,05\% sont monospécifiques. La feuille (33\%) a été l'organe végétal le plus utilisé et la décoction (69\%) le mode de préparation le plus pratiqué. La voie orale (93\%) a été la voie d'administration la plus employée. La caractérisation phytochimique des espèces végétales a révélé la présence des alcaloïdes, des tanins, des flavonoïdes, des saponosides, des stérols et des polyterpènes. Cette étude a permis de montrer que l'hypertension artérielle est connue des tradithérapeutes du département de Divo. 
Mots clés : Plantes médicinales, Hypertension artérielle, Ethnobotanique, Tradithérapeutes, Divo

\title{
Ethnobotanical Study of Plants Used in Traditional Medicine in the Treatment of Arterial Hypertension in Peoples of Divo Department, (Center-West, Côte d'Ivoire)
}

\author{
Wangny Akesse Ackah Stephane, \\ Ouattara Tieba Victor, \\ Université Félix Houphouët-Boigny, Laboratoire de Physiologie Végétale, \\ UFR Biosciences, Abidjan, Côte d'Ivoire \\ Abrou N'Gouan Emmanuel Joel,
N'guessan Koffi, \\ Université Félix Houphouët-Boigny, Laboratoire de Botanique, \\ UFR Biosciences, Abidjan, Côte d'Ivoire
}

\begin{abstract}
In order to know the medicinal plants and their traditional uses on arterial hypertension, an Ethnobotanical study was carried out in the department of Divo (Central West Forestry Region). Ethnobotanical surveys made it possible to visit 21 villages and to meet, during semi-structured interviews, 105 traditional healers, regardless of age, sex and level of education. This study showed that the surveyed populations employed 52 plant species belonging to 30 botanical families, to treat arterial hypertension. Among these families, the Fabaceae with $17.31 \%$ of the plants surveyed were the most represented. These taxa, dominated by trees $(50 \%)$, belong mainly to both the Guineo-Congolese region and the Sudano-Zambézienne region (GCSZ) with $36.54 \%$ of the species collected. The medicinal plants identified were used to prepare 154 medicinal recipts, of which 151 or $98.05 \%$ are monospecific receipts. The leaf (33\%) was the most used plant organ and the decoction $(69 \%)$ was the most popular method of preparation. The oral route (93\%) was the most used route of administration. Phytochemical characterization of plant species revealed the presence of alkaloids, tannins, flavonoids, saponosides, sterols and polyterpenes. This study showed traditional therapists in Divo department knew that arterial hypertension.
\end{abstract}


Keywords: Medicinal plants, Arterial Hypertension, Ethnobotanic, Traditional healers, Divo

\section{Introduction}

L'hypertension artérielle est une maladie cardiovasculaire. En 2000, la prévalence globale de l'hypertension dans la population mondiale adulte était estimée à $26,4 \%$ (26,6\% chez les hommes et $26,1 \%$ chez des femmes). Parmi les 972 millions d'individus hypertendus, 333 millions, soit 34,33\% proviennent des pays « développés » et 639 millions, soit $65,7 \%$ sont issus des pays « en développement ». Le nombre d'individus hypertendus d'ici à 2025 pourrait augmenter de $60 \%$ pour atteindre 1,56 milliard (Kearney et al., 2005). D'après les récentes statistiques, dans le monde, l'hypertension tuerait 9,4 millions de personnes par an. En Afrique, elle constitue un problème de santé publique avec une fréquence en population comprise entre 15 et $40 \%$, une fréquence hospitalière comprise entre 30 et $70 \%$ (Diallo et al., 2010). En Afrique de l'Ouest, le taux de prévalence avoisine les $20 \%$. La prévalence de l'hypertension artérielle en Côte d'Ivoire est généralement estimée entre 8$12 \%$. Elle concernait en $2000,13,90 \%$ de la population avec $21 \%$ pour la seule ville d'Abidjan (Koffi, 2007). Depuis environ une dizaine d'années, 40 à 50\% des patients de l'Institut de Cardiologie d'Abidjan présentent cette pathologie et $14 \%$ de la population ivoirienne en souffrent (Anonyme, 2003). Aujourd'hui, ce taux se situe autour de 33,4\%, pour des personnes de 25 ans et plus. Ce taux de prévalence élevé est inquiétant. Actuellement, la thérapie de l'hypertension repose sur l'utilisation de remèdes onéreux, souvent mal tolérés, qui nécessitent un traitement prolongé et non curable (Gentilini, 1993). La présente étude a été menée afin d'identifier les plantes hypotensives utilisées par les peuples du département de Divo et contribuer à mieux les valoriser par des recherches ethnobotaniques et phytochimiques.

\section{Matériel et méthodes \\ Milieu d'étude}

Située dans la partie occidentale de l'Afrique, la Côte d'Ivoire couvre une superficie de $322462 \mathrm{Km}^{2}$. L'étude a été réalisée dans le Centre-Ouest forestier du pays, dans la Région du Loh-Djiboua et plus précisément dans le département de Divo. Le département de Divo, avec une superficie de 3577 $\mathrm{km}^{2}$, est compris entre $5^{\circ} 40^{\prime} 00^{\prime}$ " et $6^{\circ} 10^{\prime} 00^{\prime}$ ' de latitude Nord, et entre $5^{\circ} 00^{\prime} 00^{\prime}$ et $5^{\circ} 30^{\prime} 00^{\prime}$ " de longitude Ouest. Il compte sept (07) Souspréfectures dont les chefs-lieux sont Chiépo, Didoko, Divo, Hiré, Ogoudou, Nébo et Zégo (Figure 1). Le département de Divo compte 380.220 habitants (Anonyme, 2016). Aux côtés des autochtones Dida, existe une communauté d'allochtones, en provenance de toutes les régions de la Côte d'Ivoire et une 
communauté d'allogènes, originaires pour la plupart de la sous-région Ouestafricaine.

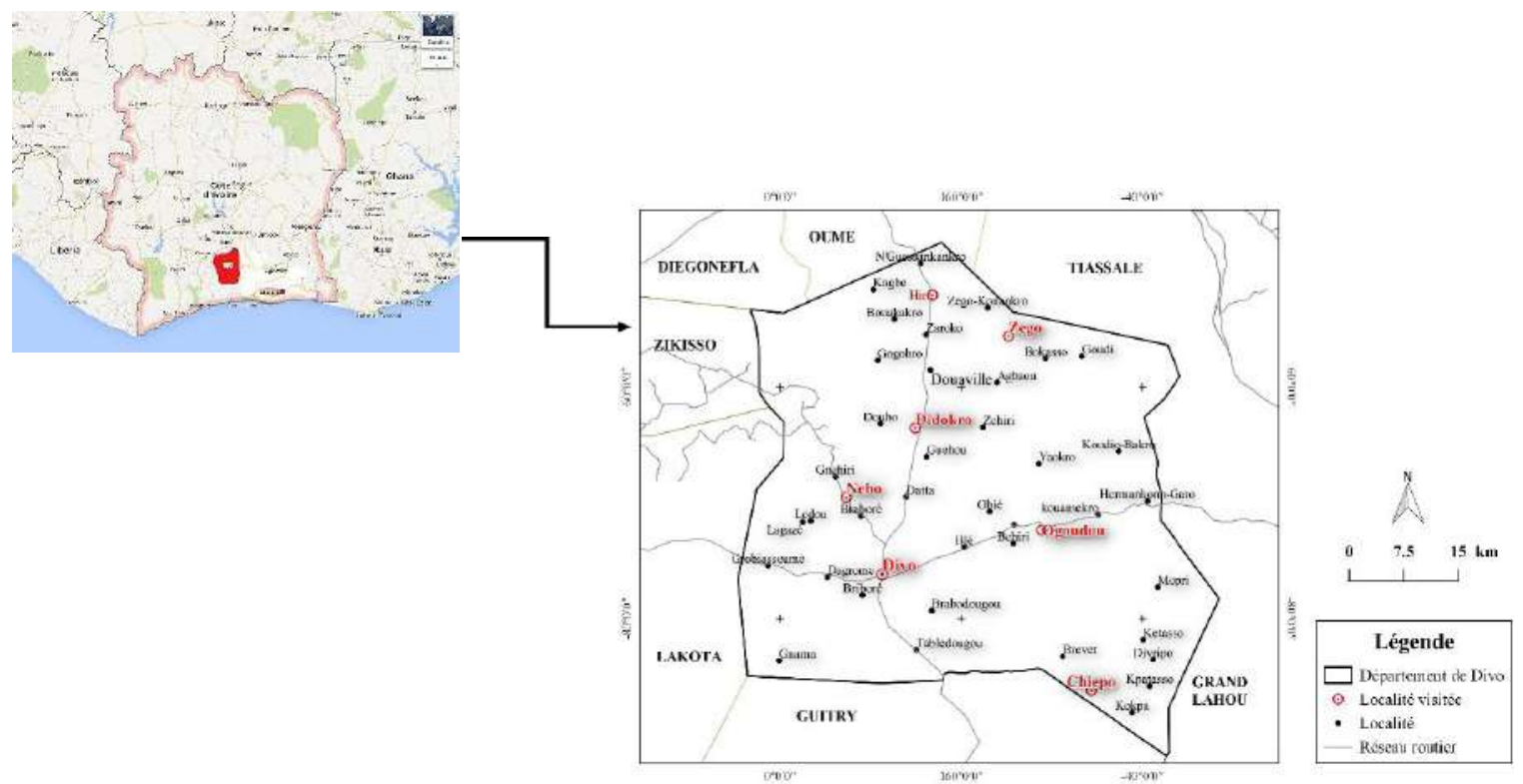

Figure 1: Situation géographique du Département de Divo Source: Google map Modifiée par Wangny, 2018

\section{Matériel végétal}

Le matériel végétal a été constitué par l'ensemble des espèces végétales (arbres, arbustes, arbrisseaux, herbes et lianes) répertoriées et récoltées dans le département de Divo.

\section{Matériel chimique}

Pour effectuer le criblage phytochimique, divers réactifs chimiques classiques (Bornstraëgen, Burchard, Chlorure ferrique, Dragendorff, Liebermann, Stiasny) ont été utilisés.

\section{Méthodes d'étude}

\section{Enquêtes ethnobotaniques}

Les investigations sur l'usage traditionnel des plantes servant à traiter l'hypertension artérielle ont été effectuées par des approches ethnobotaniques auprès des acteurs de la médecine traditionnelle. Ces enquêtes ethnobotaniques ont été menées dans sept (07) sous-préfectures du département de Divo. Les sous-préfectures ont constitué les strates. Trois villages par strate ont été visités soit au total vingt et un sites prospectés. L'entretien semi-structuré a été privilégié. Les entretiens ont été réalisés selon deux cas, soit, au cours d'une sortie en brousse comme le suggère Cunningham 
(2002) et Béné et al. (2016) ; soit, si l'informateur est trop âgé ou occupé, la méthode de Diatta et al. (2013) a été appliquée. Au cours des entretiens, le profil (sexe, âge et niveau de scolarisation) des enquêtés et les données ethnobotaniques (les différentes parties employées comme drogues, leurs usages traditionnels, leurs modes de préparation et d'administration) ont été relevés. Les échantillons de plantes récoltés ont été identifiés et ont servi à la réalisation d'un herbier. L'identification des espèces a été faite à l'herbier du Centre National de Floristique (CNF), de l'Université Félix HOUPHOUËTBOIGNY (Côte d'Ivoire). La terminologie cladistique, selon APG IV (2016) a été utilisée pour l'identification des différentes espèces de plantes.

\section{Préparation des extraits aqueux}

Les différentes drogues (écorce de tige, feuilles et graine) de six espèces de plantes (Centella asiatica, Ficus thonningii, Ricinodendron heudelotii, Solenostemon monostachyus, Spondias mombin, Theobroma cacao), traditionnellement utilisées contre l'hypertension artérielle ont été préalablement lavées puis séchées à l'ombre dans un local aéré pendant 2 semaines. Chaque organe de plante séché a été rendu en poudre fine en utilisant un broyeur électrique, puis conservé dans un bocal $\mathrm{sec}$ et hermétiquement fermé pour éviter toute contamination de moisissures. Sur cette poudre, une extraction aqueuse a été effectuée, selon le protocole de Aouinty et al. (2006). Une quantité de $100 \mathrm{~g}$ de poudre de chaque drogue est diluée dans un litre d'eau distillée préalablement portée à ébullition, puis laissée refroidir sous agitation magnétique pendant 30 minutes. Le mélange obtenu a été filtré à l'aide du papier Wattman $(3 \mathrm{~mm})$. Le filtrat récupéré représente une solution initiale à $100 \mathrm{~g} / \mathrm{l}$ soit $10 \%$. Le filtrat a été concentré par évaporation dans une étuve portée à $40^{\circ} \mathrm{C}$ pendant 48 heures, jusqu'à obtention d'un résidu sec dont la quantité est exprimée en mg.

\section{Caractérisation des groupes chimiques}

Les extraits aqueux de ces six espèces de plantes ont été caractérisés selon les méthodes décrites par Ronchetti et Russo (1971), Hegnauer (1973), Wagner (1983), Bekro et al. (2007). Le détail concernant les réactifs utilisés et les réactions caractéristiques des groupes chimiques sont contenus dans le tableau 1. 
Tableau 1: Réactifs et tests de caractérisation des groupes chimiques

\begin{tabular}{|c|c|c|c|}
\hline \multicolumn{2}{|c|}{ Composés chimiques } & \multirow{2}{*}{$\begin{array}{l}\text { Réactifs } \\
\text { Stiasny }\end{array}$} & \multirow{2}{*}{$\begin{array}{l}\text { Réaction indiquant que le test } \\
\text { est positif } \\
\text { Précipité brun-verdâtre }\end{array}$} \\
\hline \multirow[b]{2}{*}{ Tanins } & Catéchiques & & \\
\hline & Galliques & $\begin{array}{l}\text { Stiasny et } \\
\text { chlorure ferrique }\end{array}$ & Précipité bleu-noirâtre \\
\hline \multicolumn{2}{|c|}{ Stérols et polyterpènes } & Liebermann & $\begin{array}{l}\text { Précipité pourpre ou violet qui } \\
\text { vire au bleu puis au vert }\end{array}$ \\
\hline \multicolumn{2}{|c|}{ Flavonoïdes } & Cyanidine & $\begin{array}{l}\text { Coloration rose-orangé ou } \\
\text { violacée }\end{array}$ \\
\hline \multicolumn{2}{|c|}{ Polyphénols } & Chlorure ferrique & $\begin{array}{l}\text { Coloration bleu-noirâtre ou } \\
\text { verte } \pm \text { foncée }\end{array}$ \\
\hline \multirow{2}{*}{\multicolumn{2}{|c|}{ Alcaloïdes }} & Burchard & $\begin{array}{l}\text { Précipité ou coloration brun- } \\
\text { rougeâtre }\end{array}$ \\
\hline & & Dragendorff & Précipité ou coloration orangée \\
\hline \multicolumn{2}{|c|}{ Quinones } & Bornstraëgen & $\begin{array}{l}\text { Coloration allant du rouge au } \\
\text { violet }\end{array}$ \\
\hline \multicolumn{2}{|c|}{ Saponosides } & $\begin{array}{l}\text { Agitation du tube } \\
\text { contenant l'extrait }\end{array}$ & $\begin{array}{l}\text { Mousse persistante supérieure à } \\
1 \mathrm{~cm} \text { de hauteur }\end{array}$ \\
\hline
\end{tabular}

\section{Résultats}

Sites visités et caractéristiques

\section{des enquêtés}

Les enquêtes ethnobotaniques ont été effectuées dans 21 villages répartis en 07 strates. Au total, 105 praticiens de santé ont été visités dont 59 hommes soit $56,19 \%$ et 46 femmes soit $43,81 \%$. Par ailleurs les personnes non scolarisées $(53,33 \%)$ ont été majoritaires (Tableau 2).

L'âge des guérisseurs interrogés a varié de 22 à 75 ans. Mais la tranche d'âge de 35-50 ans avec $61,90 \%$ a été la plus représentée (Tableau 3)

Tableau 2: Nombre et proportion des enquêtés par sexe et niveau de scolarisation

\begin{tabular}{|c|c|c|c|c|c|c|c|c|c|}
\hline Paramètres & & $\begin{array}{c}\text { Strate } \\
1\end{array}$ & $\begin{array}{c}\text { Strate } \\
2\end{array}$ & $\begin{array}{c}\text { Strate } \\
\mathbf{3}\end{array}$ & $\begin{array}{c}\text { Strate } \\
4\end{array}$ & $\begin{array}{c}\text { Strate } \\
5\end{array}$ & $\begin{array}{c}\text { Strate } \\
6\end{array}$ & $\begin{array}{c}\text { Strate } \\
7\end{array}$ & Totaux \\
\hline \multirow[b]{2}{*}{ Hommes } & Effectif & 9 & 8 & 8 & 9 & 11 & 8 & 6 & 59 \\
\hline & $\begin{array}{l}\text { Proportion } \\
(\%)\end{array}$ & 60 & 53,33 & 53,33 & 60 & 73,33 & 53,33 & 40 & 56,19 \\
\hline \multirow[b]{2}{*}{ Femmes } & Effectif & 6 & 7 & 7 & 6 & 4 & 7 & 9 & 46 \\
\hline & $\begin{array}{l}\text { Proportion } \\
(\%)\end{array}$ & 40 & 46,67 & 46,67 & 40 & 26,67 & 46,67 & 60 & 43,81 \\
\hline \multirow[b]{2}{*}{ Scolarisés } & Effectif & 9 & 9 & 8 & 9 & 5 & 4 & 4 & 48 \\
\hline & $\begin{array}{l}\text { Proportion } \\
(\%)\end{array}$ & 60 & 60 & 53,33 & 60 & 33,33 & 26,67 & 33,33 & 46,67 \\
\hline \multirow[b]{2}{*}{ Analphabètes } & Effectif & 6 & 6 & 7 & 6 & 10 & 11 & 8 & 54 \\
\hline & $\begin{array}{l}\text { Proportion } \\
(\%)\end{array}$ & 9,09 & 9,09 & 11,6 & 9,09 & 23,07 & 29,2 & 19,35 & 53,33 \\
\hline
\end{tabular}


Tableau 3: Nombre et proportion des enquêtés par sexe

\begin{tabular}{|c|c|c|c|c|c|c|c|c|c|}
\hline Classe d'âge & & $\begin{array}{c}\text { Strate } \\
1 \\
\end{array}$ & $\begin{array}{c}\text { Strate } \\
2 \\
\end{array}$ & $\begin{array}{c}\text { Strate } \\
\mathbf{3} \\
\end{array}$ & $\begin{array}{c}\text { Strate } \\
4 \\
\end{array}$ & $\begin{array}{c}\text { Strate } \\
5 \\
\end{array}$ & $\begin{array}{c}\text { Strate } \\
6 \\
\end{array}$ & $\begin{array}{c}\text { Strate } \\
7 \\
\end{array}$ & Totaux \\
\hline \multirow[b]{2}{*}{ 22-34 ans } & Effectif & 1 & 1 & 2 & 1 & 3 & 1 & 2 & 11 \\
\hline & $\begin{array}{l}\text { Proportion } \\
(\%)\end{array}$ & 6,67 & 6,67 & 13,33 & 6,67 & 20 & 6,67 & 13,33 & 10,48 \\
\hline \multirow[b]{2}{*}{$35-50$ ans } & Effectif & 9 & 11 & 9 & 9 & 8 & 10 & 9 & 65 \\
\hline & $\begin{array}{l}\text { Proportion } \\
(\%)\end{array}$ & 60 & 73,33 & 60 & 60 & 53,33 & 66,67 & 60 & 61,90 \\
\hline \multirow[b]{2}{*}{$51-75$ ans } & Effectif & 5 & 3 & 4 & 5 & 4 & 4 & 4 & 29 \\
\hline & $\begin{array}{l}\text { Proportion } \\
(\%)\end{array}$ & 33,33 & 20 & 26,67 & 33,33 & 26,67 & 26,67 & 26,67 & 27,62 \\
\hline
\end{tabular}

\section{Caractéristeques botaniques}

L'inventaire floristique a permis d'identifier 52 espèces de plantes réparties en 50 genres et 30 familles botaniques (Tableau 4). Ces espèces ont été toutes des Spermaphytes. Les Eudicotylédones avec 38 espèces soit $73,07 \%$ ont représenté la classe la plus dominante. Les Fabaceae avec 09 espèces soit $17,31 \%$ ont été la famille majoritairement citée. Les plantes médicinales inventoriées ont été regroupées en 07 types biologiques. Les Phanérophytes, avec $82,69 \%$ ont été les types biologiques les plus utilisés. Parmi ces Phanérophytes, les microphanérophytes avec 51,92\% ont été les plus représentés. Toutes les espèces identifiées ont appartenu à 04 types phytogéographiques avec une dominance des taxons communs à la région Guinéo-Congolaise et Soudano-Zambézienne (GC-SZ) comptant 19 espèces soit 36,54\%. Les taxons de la région Guinéo-congolaise (GC) avec 18 espèces soit $34,62 \%$ ont été secondairement représentés. La majorité des plantes recensées étaient des arbres avec 26 espèces soit 50\% (Figure 2).

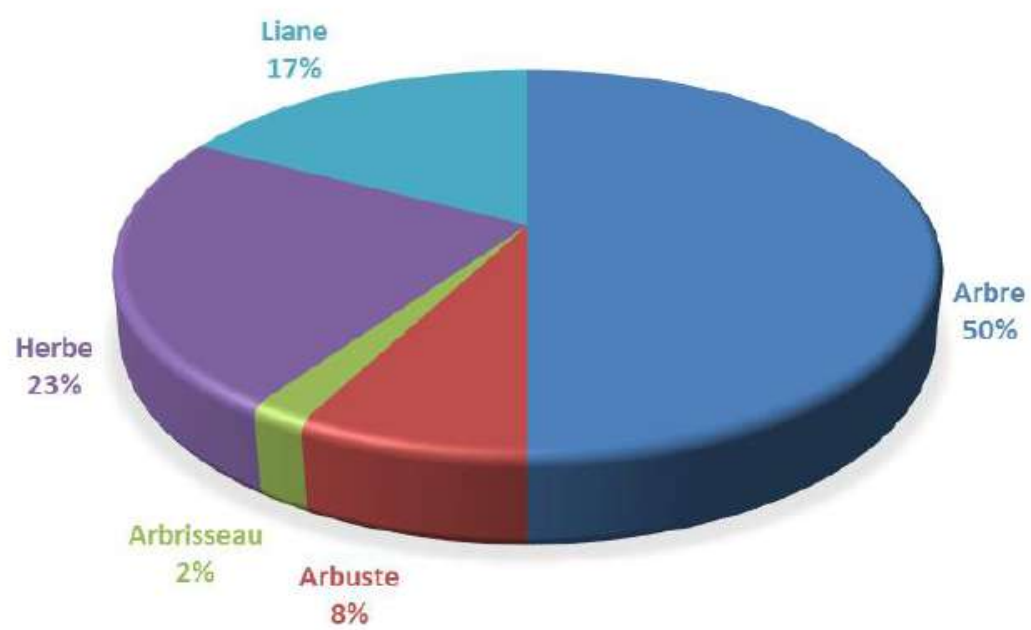

Figure 2: Spectre des différents types morphologiques des espèces de plantes répertoriées 
Tableau 4: Caractères botaniques et ethnomédicinales des plantes hypotensives recensées

\begin{tabular}{|c|c|c|c|c|c|c|c|c|c|}
\hline Taxons & Famille & $\mathbf{C l}$ & T. M & T. B & T. $\mathbf{P}$ & P. U & M. P & F. M & M. A \\
\hline Adansonia digitata Lin. & Malvaceae & Eudic & $\mathrm{Ar}$ & $\mathrm{mP}$ & SZ & Feuille & Décoction & Décocté & Boisson \\
\hline Albizia lebbeck (L.) Benth. & Fabaceae & Eudic & $\mathrm{Ar}$ & $\mathrm{Mp}$ & $\begin{array}{l}\text { GC- } \\
\text { SZ }\end{array}$ & Ecorce de tige & Macération & Macéré & Boisson \\
\hline Allium sativum L. & Liliaceae & Mon & Her & Hém & I & Bulbe & Décoction & Décocté & Boisson \\
\hline Aloe vera (L.) Burm. F & Liliaceae & Mon & Her & Hém & I & Feuille & Infusion & Infusé & Boisson \\
\hline Anacardium occidentale L. & Anacardiaceae & Eudic & $\mathrm{Ar}$ & $\mathrm{Mp}$ & I & Ecorce de tige & Macération & Macéré & Boisson \\
\hline Annona muricata $\mathrm{L}$. & Annonaceae & Paléod & $\mathrm{Ar}$ & $\mathrm{Mp}$ & GC & Feuille & Décoction & Décocté & Boisson \\
\hline Annona senegalensis Pers. & Annonaceae & Paléod & Art & $\mathrm{Np}$ & SZ & Feuille & Décoction & Décocté & Boisson \\
\hline Bambusa vulgaris Wendl.ex Nees & Poaceae & Mon & Her & Hém & $\mathrm{I}$ & Feuille & Décoction & Décocté & Boisson \\
\hline Bridelia grandis Pierre ex Hutch. & Phyllanthaceae & Eudic & $\mathrm{Ar}$ & $\mathrm{mP}$ & GC & Ecorce de tige & Décoction & Décocté & Boisson \\
\hline Caesalpinia bonduc (L.) Roxb. & Fabaceae & Eudic & Lian & $\mathrm{Mp}$ & GC & Racine & $\begin{array}{l}\text { Macération } \\
(\mathrm{St})\end{array}$ & Macéré & Boisson \\
\hline Centella asiatica (Linn.) Urb. & Apiaceae & Eudic & Her & $\mathrm{Ch}$ & $\mathrm{I}$ & Plante entière & Décoction & Décocté & Boisson \\
\hline Citrus sinensis (L.) Osbeck & Rutaceae & Eudic & $\mathrm{Ar}$ & $\mathrm{Mp}$ & $\mathrm{I}$ & Fruit & Expression & Jus & Badigeonnage \\
\hline Clerodendrum inerme (L.) & Lamiaceae & Eudic & Lian & $\mathrm{Mp}$ & I & Rameau feuillé & Décoction & Décocté & Boisson \\
\hline
\end{tabular}




\begin{tabular}{|c|c|c|c|c|c|c|c|c|c|}
\hline Gaertn. & & & & & & & & & \\
\hline Cola nitida (Vent) Schott. Endl. & Malvaceae & Eudic & $\mathrm{Ar}$ & $\mathrm{mP}$ & GC & Fruit & Décoction & Décocté & Boisson \\
\hline *Combretum paniculatum Vent. & Combretaceae & Eudic & Liane & $\mathrm{Mp}$ & $\begin{array}{l}\text { GC- } \\
\text { SZ }\end{array}$ & Feuille & Décoction & Décocté & $\begin{array}{l}\text { Bain vapeur } \\
\text { et Boisson }\end{array}$ \\
\hline Costus afer Ker-Gawl. & Zingiberaceae & Mon & Her & $\mathrm{Np}$ & GC & Rhizome & Mastication & Jus & Boisson \\
\hline Crinum jagus (Thomps.) Dandy & Amaryllidaceae & Mon & Her & G & $\begin{array}{l}\text { GC- } \\
\text { SZ }\end{array}$ & Bulbe & Décoction & Décocté & Boisson \\
\hline Cymbopogon citratus (DC.) Stapf & Poaceae & Mon & Her & Hém & $\begin{array}{l}\text { GC- } \\
\text { SZ }\end{array}$ & Feuille & Infusion & Infusé & Boisson \\
\hline Daniellia oliveri Hutch. \& Dalz. & Fabaceae & Eudic & $\mathrm{Ar}$ & $\mathrm{mP}$ & $\mathrm{SZ}$ & Feuille & Décoction & Décocté & Boisson \\
\hline Ficus thonningii Blume & Moraceae & Eudic & $\mathrm{Ar}$ & $\mathrm{Mp}$ & $\begin{array}{l}\text { GC- } \\
\text { SZ }\end{array}$ & Ecorce de tige & Décoction & Décocté & Boisson \\
\hline Garcinia kola Heckel & Clusiaceae & Eudic & $\mathrm{Ar}$ & $\mathrm{mP}$ & GC & Graine & Mastication & Mastiqué & Absorption \\
\hline Icacina mannii Oliv. & Icacinaceae & Eudic & Lian & $\mathrm{Mp}$ & GC & Tubercule & Décoction & Décocté & Boisson \\
\hline Kigelia africana (Lam.) Benth. & Bignoniaceae & Eudic & $\mathrm{Ar}$ & $\mathrm{Mp}$ & $\begin{array}{l}\text { GC- } \\
\text { SZ }\end{array}$ & Ecorce de tige & Pétrissage & $\begin{array}{l}\text { Poudre } \\
\text { (AC) }\end{array}$ & Absorption \\
\hline Lantana Camara L. & Verbenaceae & Eudic & Lian & $\mathrm{Mp}$ & GC & Rameau feuillé & Infusion & Infusé & Boisson \\
\hline Manniophyton fulvum Müll. Arg. & Euphorbiaceae & Eudic & Lian & $\mathrm{Mp}$ & GC & Rameau feuillé & Décoction & Décocté & Boisson \\
\hline Manotes longiflora Baker & Connaraceae & Eudic & Lian & $\mathrm{Mp}$ & GC & Feuille & Décoction & Décocté & Boisson \\
\hline Morinda lucida Benth. & Rubiaceae & Eudic & $\mathrm{Ar}$ & $\mathrm{Mp}$ & $\begin{array}{l}\text { GC- } \\
\text { SZ }\end{array}$ & Feuille & Décoction & Décocté & Boisson \\
\hline
\end{tabular}




\begin{tabular}{|c|c|c|c|c|c|c|c|c|c|}
\hline Moringa oleifera Lam & Moringaceae & Eudic & $\mathrm{Ar}$ & $\mathrm{Mp}$ & $\begin{array}{l}\text { GC- } \\
\text { SZ }\end{array}$ & Feuille & Décoction & Décocté & Boisson \\
\hline Ocimum gratissimum $\mathrm{L}$. & Lamiaceae & Eudic & Art & $\mathrm{Np}$ & $\begin{array}{l}\text { GC- } \\
\text { SZ }\end{array}$ & Rameau feuillé & Décoction & Décocté & Boisson \\
\hline $\begin{array}{l}\text { Opilia celtidifolia (Guill. Et Perr.) } \\
\text { Endl. }\end{array}$ & Opiliaceae & Eudic & Lian & $\mathrm{Mp}$ & $\begin{array}{l}\text { GC- } \\
\text { SZ }\end{array}$ & Feuille & Décoction & Décocté & Boisson \\
\hline Palisota hirsuta (Thunb.) Engl. & Commelinaceae & Mon & Her & $\mathrm{Np}$ & GC & Plante entière & Décoction & Décocté & Boisson \\
\hline $\begin{array}{c}\text { Parkia biglobosa (Jacq.) R.Br. Ex } \\
\text { G.Don }\end{array}$ & Fabaceae & Eudic & $\mathrm{Ar}$ & $\mathrm{Mp}$ & SZ & Fruit & Pilage & Soupe & Absorption \\
\hline $\begin{array}{l}\text { *Pericopsis laxiflora (Benth) } \\
\text { Meeuv. }\end{array}$ & Fabaceae & Eudic & $\mathrm{Ar}$ & $\mathrm{Mp}$ & $\begin{array}{l}\text { GC- } \\
\text { SZ }\end{array}$ & Rameau feuillé & Décoction & Décocté & $\begin{array}{l}\text { Bain vapeur } \\
\text { et Boisson }\end{array}$ \\
\hline Persea americana Mill. & Lauraceae & Eudic & $\mathrm{Ar}$ & $\mathrm{Mp}$ & I & Feuille sèche & Décoction & Décocté & Boisson \\
\hline $\begin{array}{c}\text { Phyllanthus amarus Schum. Et } \\
\text { Thonn. }\end{array}$ & Phyllanthaceae & Eudic & Ars & $\mathrm{Np}$ & $\mathrm{GC}$ & Plante entière & Décoction & Décocté & Boisson \\
\hline Piper guineense Schum. et Thonn. & Piperaceae & Paléod & Lian & $\mathrm{Mp}$ & GC & Rameau feuillé & Décoction & Décocté & Boisson \\
\hline $\begin{array}{c}\text { Ricinodendron heudelotii (Baille) } \\
\text { Pierre }\end{array}$ & Euphorbiaceae & Eudic & $\mathrm{Ar}$ & $\mathrm{mP}$ & $\mathrm{GC}$ & Graine & Broyage & Soupe & Absorption \\
\hline Schrankia leptocarpa DC. & Fabaceae & Eudic & Lian & $\mathrm{Np}$ & GC & Rameau feuillé & Décoction & Décocté & Boisson \\
\hline $\begin{array}{l}\text { Scleria depressa (C.B.CI.) } \\
\text { Nelmes }\end{array}$ & Cyperaceae & Mon & Her & $\mathrm{G}$ & $\begin{array}{l}\text { GC- } \\
\text { SZ }\end{array}$ & Rameau feuillé & Broyage & Pâte & Purge \\
\hline $\begin{array}{c}\text { *Securidaca longepedunculata } \\
\text { Fresen. }\end{array}$ & Polygalaceae & Eudic & $\mathrm{Ar}$ & $\mathrm{Mp}$ & SZ & Feuille & Décoction & Décocté & $\begin{array}{l}\text { Bain vapeur } \\
\text { et Boisson }\end{array}$ \\
\hline
\end{tabular}




\begin{tabular}{|c|c|c|c|c|c|c|c|c|c|}
\hline Senna alata $\mathrm{L}$. & Fabaceae & Eudic & Art & $\mathrm{Np}$ & $\mathrm{GC}$ & Feuille & Décoction & Décocté & Boisson \\
\hline Senna occidentalis L. & Fabaceae & Eudic & Art & $\mathrm{Np}$ & $\begin{array}{l}\text { GC- } \\
\text { SZ }\end{array}$ & Feuille & Décoction & Décocté & Boisson \\
\hline $\begin{array}{l}\text { Solenostemon monostachyus } \\
\text { (P.Beauv.) Briq. Subsp. }\end{array}$ & Lamiaceae & Mon & Her & Th & $\begin{array}{l}\text { GC- } \\
\text { SZ }\end{array}$ & Feuille & Décoction & Décocté & Boisson \\
\hline Spondias mombin L. & Anacardiaceae & Eudic & $\mathrm{Ar}$ & $\mathrm{Mp}$ & $\begin{array}{l}\text { GC- } \\
\text { SZ }\end{array}$ & Ecorce de tige & Décoction & Décocté & Boisson \\
\hline Tamarindus indica Linn. & Fabaceae & Eudic & $\mathrm{Ar}$ & $\mathrm{Mp}$ & $\begin{array}{l}\text { GC- } \\
\text { SZ }\end{array}$ & Ecorce de tige & Décoction & Décoction & Boisson \\
\hline Theobroma cacao L. & Malvaceae & Eudic & $\mathrm{Ar}$ & $\mathrm{Mp}$ & I & Graine & Macération & Macéré & Boisson \\
\hline Tridax procumbens L. & Asteraceae & Eudic & Her & $\mathrm{Np}$ & $\begin{array}{l}\text { GC- } \\
\text { SZ }\end{array}$ & Plante entière & Décoction & Décocté & Boisson \\
\hline Uapaca guineensis Müll. Arg. & Phyllanthaceae & Eudic & $\mathrm{Ar}$ & $\mathrm{mP}$ & $\mathrm{GC}$ & Ecorce de tige & Décoction & Décocté & Boisson \\
\hline Vernonia colorata (Will.) Drake & Asteraceae & Eudic & Art & $\mathrm{Mp}$ & $\begin{array}{l}\text { GC- } \\
\text { SZ }\end{array}$ & Racine & Décoction & Décocté & Boisson \\
\hline Voacanga africana Staff. & Apocynaceae & Eudic & Ar & $\mathrm{Mp}$ & $\mathrm{GC}$ & Ecorce de tige & Décoction & Décocté & Boisson \\
\hline $\begin{array}{l}\text { Xylopia aethiopca (Dunal.) A. } \\
\text { Roch. }\end{array}$ & Annonaceae & Paléod & $\mathrm{Ar}$ & $\mathrm{Mp}$ & $\begin{array}{l}\text { GC- } \\
\text { SZ }\end{array}$ & Fruit & Pulvérisation & Poudre & Boisson \\
\hline Zingiber officinale Rose. & Zingiberaceae & Mon & Her & $\mathrm{Cr}$ & I & Rhizome & Pilage & Jus & Boisson \\
\hline
\end{tabular}

Légende : $\mathrm{Cl}$ : Classe ; T.M : Type morphologique ; T.B : Type biologique ; T.P : Type phytogéographique ; P.U : Partie utilisée ; M.P : mode de préparation ; F.M : Forme médicamenteuse ; M.A : Mode d'administration ; Eudic : Eudicotylédone ; Mon : Monocotylédone ; Paléod : Paléodicotyledone ; Ar : Arbre ; Art : Arbuste ; Ars : Arbrisseau ; Her : Herbe ; Lian : Liane ; mP : Mésophanérophyte ; mp : Microphanérophyte ; np : Nanophanérophyte ; Cr : Cryptophyte ; Hém : Hémicryptophyte ; Ch : Chaméphyte ; G :Géophyte ; Th : Thérophyte ; I : Introduite ; GC : Guinéo-Congolaise ; SZ: Soudano-Zambésienne ; GC-SZ : Guinéo-Congolaise et Soudano-Zambésienne, $\left(^{*}\right)$ : Recette plurispécifique 


\section{Caractéristiques ethnomédicinales}

\section{Parties de plantes utilisées}

Le spectre des parties de plantes utilisées comme drogue a été consigné dans la figure 3. Les résultats ont révélé que les feuilles avec 33\%, ont été les parties de plantes les plus utilisées. Elles ont été suivies des écorces de tiges et des rameaux. Les parties les moins employées ont été constituées de bulbes $(4 \%)$, racines $(4 \%)$ et des tubercules $(2 \%)$.

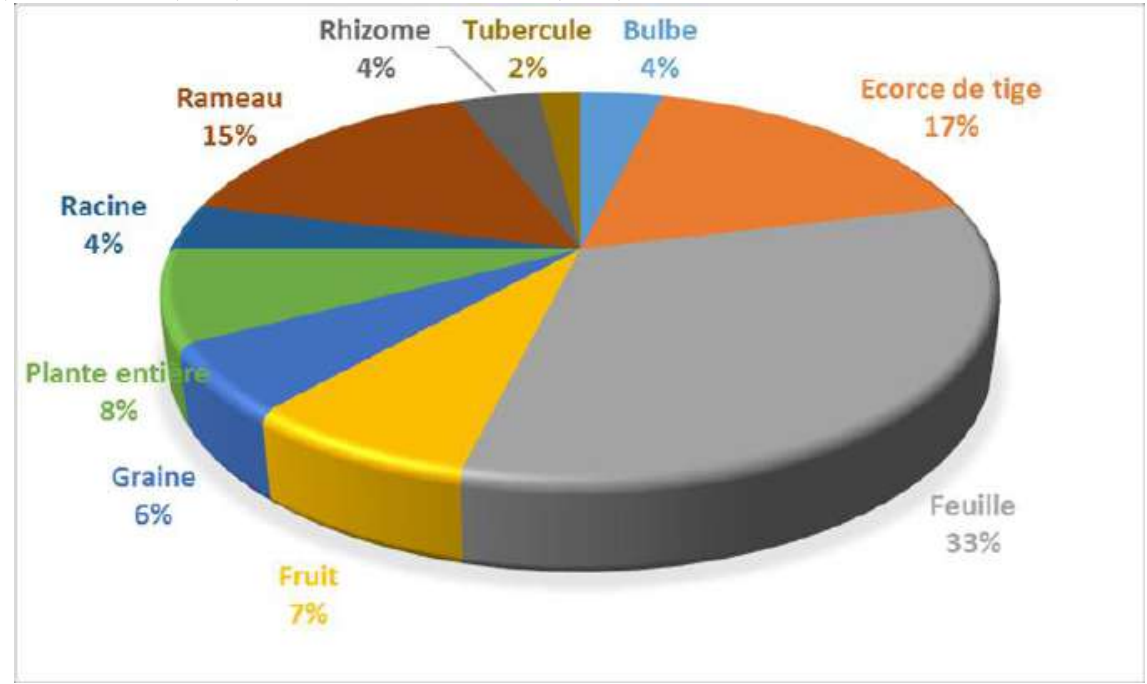

Figure 3: Spectre des différentes parties de plantes utilisées

\section{Modes de préparation et voies d'administration des recettes médicamenteuses}

La figure 4 Présente les différents modes de préparation des recettes médicamenteuses. Elles ont varié d'une plante à l'autre. Dans l'ensemble, la décoction $(69 \%)$ comme observée dans la figure 5 est le mode de préparation médicamenteuse le plus utilisé suivi de la macération (7\%) et de l'infusion (6\%). Les modes de préparation les moins proposés ont été l'expression, le pétrissage et la pulvérisation avec $2 \%$ chacun. Les recettes monospécifiques avec 98,05\% ont été prédominant (Tableau 5).

La boisson $(83 \%)$ a été le mode d'administration le plus utilisé suivi de l'absorption (7\%) et le bain vapeur avec 6\% (Figure 6). Les tradithérapeutes ont eu très peu recours au badigeonnage et la purge $(2 \%)$. La voie orale $(93 \%)$ a été la plus proposée dans le traitement de l'hypertension artérielle (Figure 7). 


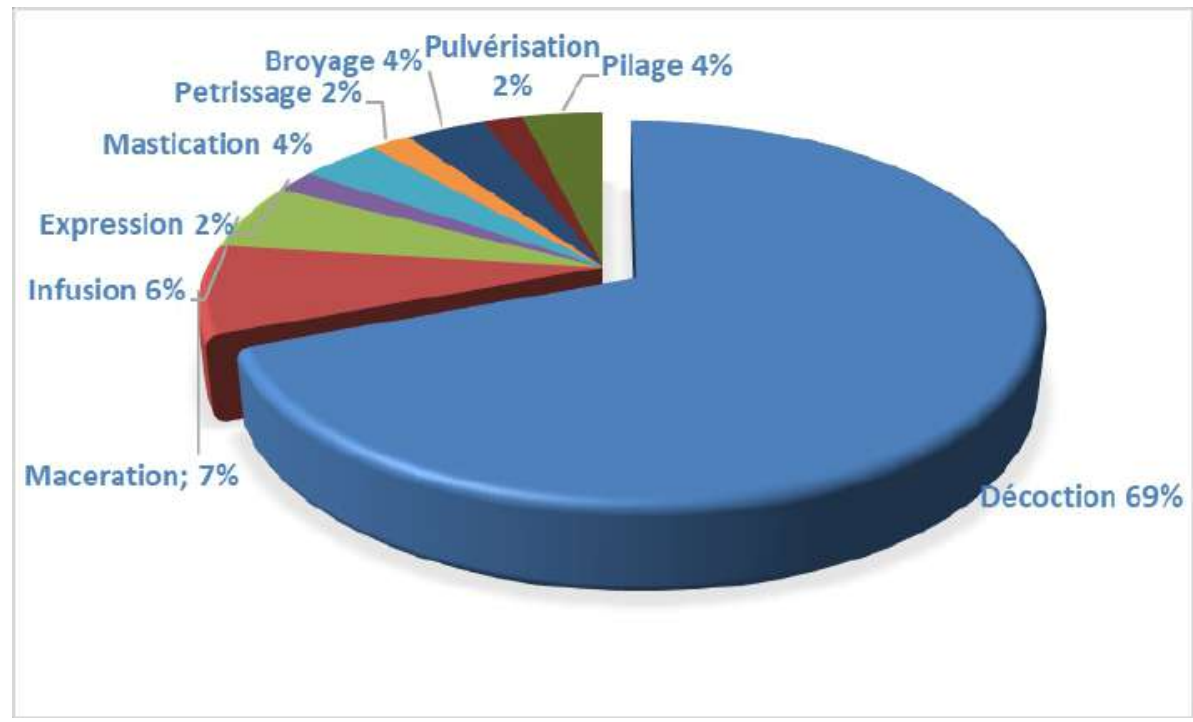

Figure 4: Spectre des différentes méthodes de preparation des recettes édicamenteuses
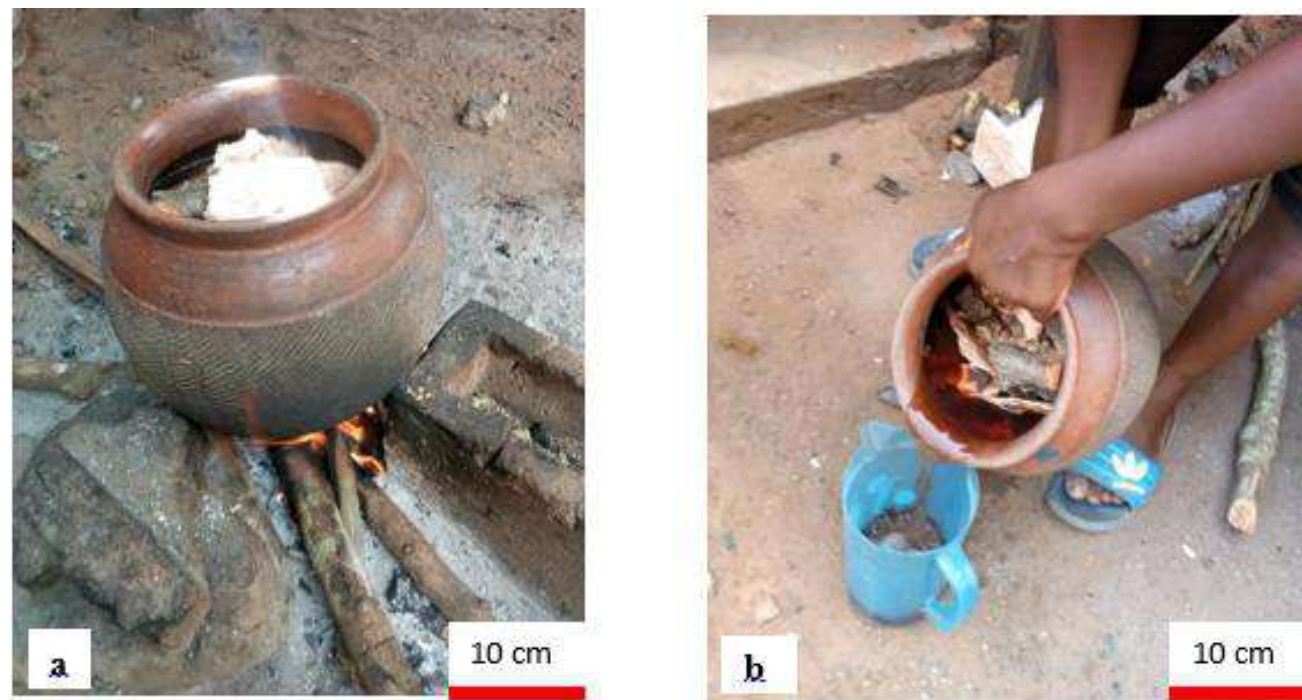

Figure 5: Réalisation d'une décoction à partir d'écorces de tige de Spondias mombin L. (nom local, Dida : Totrossou) pour le traitement de l'hypertension artérielle. $a$ : Décoction, $b$ : Décocté 


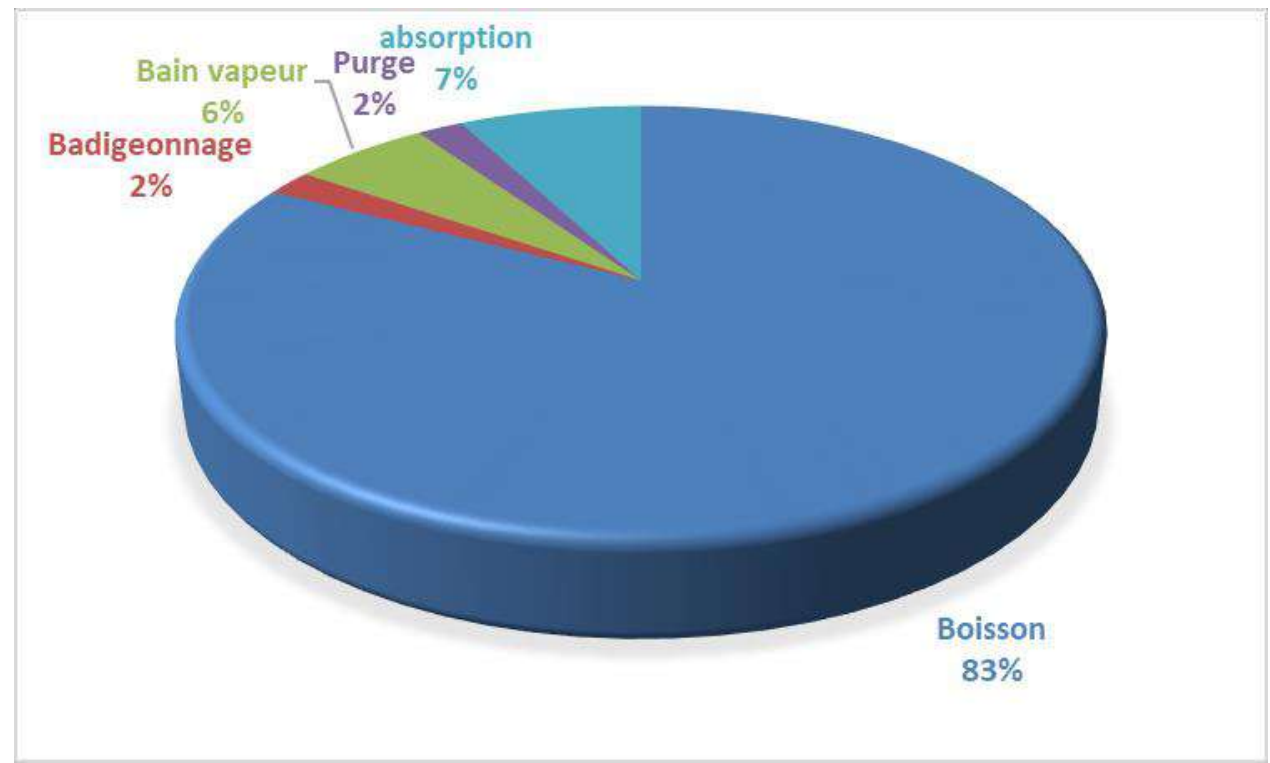

Figure 6: Spectre des différents modes d'administration des recettes médicamenteuses

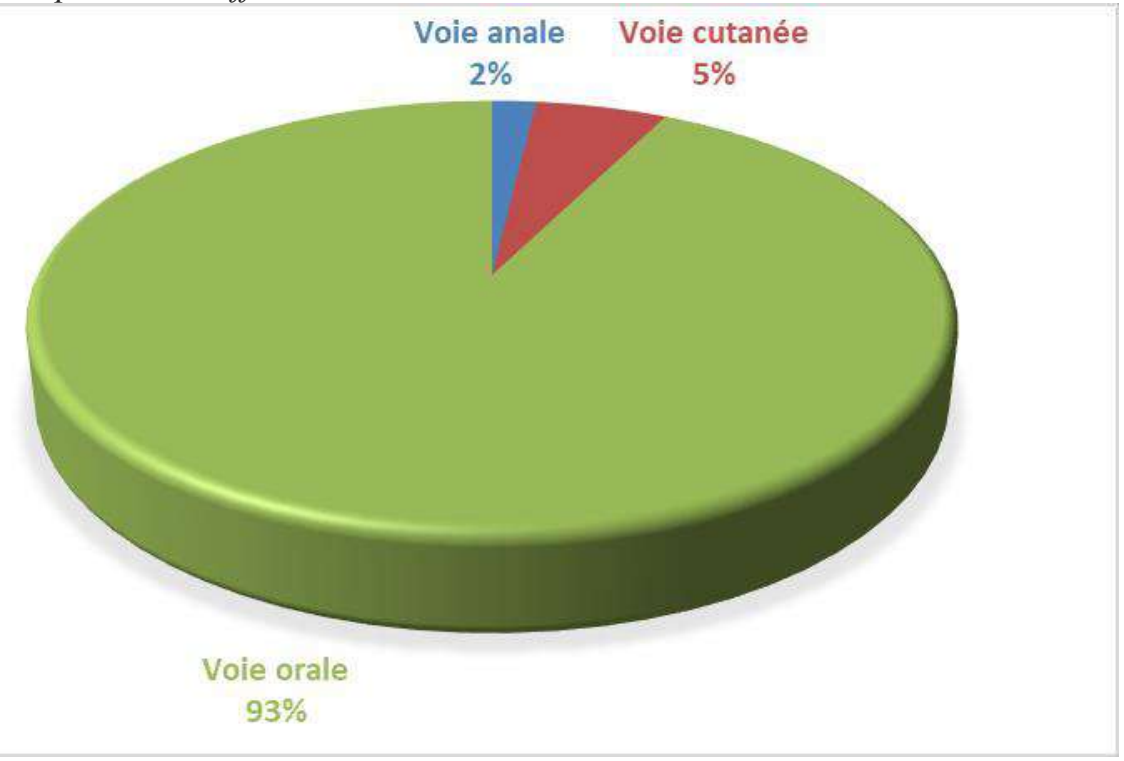

Figure 7: Spectre des différentes voies d'administration des recettes médicamenteuses

Tableau 5: Nombre et proportion du type d'association des plantes intervenant dans la recette médicamenteuse

\begin{tabular}{|c|c|c|}
\cline { 2 - 3 } \multicolumn{1}{c|}{} & Nombre & Pourcentage \\
\hline Recette monospécifique & 151 & 98,05 \\
\hline Recette plurispécifique & 3 & 1,95 \\
\hline
\end{tabular}




\section{Caractérisation phytochimique de six espèces de plantes.}

Le tableau 6 montre les tests phytochimiques effectués sur les extraits aqueux de six espèces de plantes répertoriées. Les résultats ont révélé que trois espèces de plantes contiennent tous les composés chimiques dosés à l'exception des tanins galliques. Cependant, toutes les autres espèces ont été constituées d'au moins trois composés chimiques.

Tableau 6: Caractérisation phytochimique de six espèces de plantes

\begin{tabular}{|c|c|c|c|c|c|c|c|c|c|}
\hline \multirow{3}{*}{$\begin{array}{l}\text { Extraits } \\
\text { aqueux }\end{array}$} & \multicolumn{9}{|c|}{ Composés chimiques } \\
\hline & \multirow{2}{*}{$\begin{array}{c}\text { Stérols } \\
\text { et } \\
\text { polyter- } \\
\text { pènes }\end{array}$} & \multirow[b]{2}{*}{$\begin{array}{c}\text { Polyphé } \\
\text { nols }\end{array}$} & \multirow[b]{2}{*}{$\begin{array}{l}\text { Flavono } \\
\text { ides }\end{array}$} & \multicolumn{2}{|c|}{ Tanins } & \multirow[b]{2}{*}{$\begin{array}{l}\text { Quino } \\
\text { nes }\end{array}$} & \multicolumn{2}{|c|}{ Alcaloïdes } & \multirow[b]{2}{*}{$\begin{array}{l}\text { Sapono- } \\
\text { sides }\end{array}$} \\
\hline & & & & $\begin{array}{l}\text { Catéch } \\
\text { iques }\end{array}$ & $\begin{array}{l}\text { Galli } \\
\text { ques }\end{array}$ & & A & $\mathrm{D}$ & \\
\hline Centella asiatica & + & + & + & + & - & + & + & + & ++ \\
\hline Ficus thonningii & + & - & - & - & - & + & + & + & - \\
\hline $\begin{array}{l}\text { Ricinodendron } \\
\text { heudelotii }\end{array}$ & ++ & - & $+/-$ & - & - & - & + & + & - \\
\hline $\begin{array}{l}\text { Solenostemon } \\
\text { monostachyus }\end{array}$ & + & + & + & ++ & - & + & + & + & + \\
\hline Spondias mombin & + & + & + & ++ & - & + & + & + & ++ \\
\hline Theobroma cacao & + & - & + & ++ & - & + & + & + & - \\
\hline
\end{tabular}

Légende : - : Absent ; + : présent ; ++ : abondant ; +/- : faible

\section{Discussion}

\section{Age, sexe et niveau de scolarisation des enquêtés}

Les guérisseurs interrogés sont compris majoritairement dans la tranche d'âge de 35-50 ans $(61,90 \%)$. Ce qui indique que ce sont les adultes qui s'intéressent davantage à l'art médical traditionnel. Ces résultats sont similaires à ceux de Mpondo et al. (2017) et Yapo (2017). De plus, la plupart des personnes de sexe masculin $(56,19 \%)$ ont participé à l'enquête contre $43,81 \%$ de sexe féminin. En effet, les enquêtes menées en Côte d'Ivoire par Ouattara (2006), N'guessan et al. (2011), avec respectivement $87 \%$ et $60 \%$ d'hommes et au Cameroun par Ngoule et al. (2015) avec 60,41\% d'hommes, ont montré que les tradithérapeutes de sexe masculin s'intéressent plus à l'art médical traditionnel que les femmes. D'autre part, les enquêtés sont majoritairement analphabètes $(53,33 \%)$. Ce qui implique que le niveau de scolarisation de la population locale est insuffisant.

\section{Caractéristiques botaniques}

Les plantes ligneuses ont été les plus utilisées par les peuples de ce département pour se soigner du fait de leur abondance dans leurs forêts. La proportion élevée des Eudicotylédones s'expliquerait par leur forte présence en espèces et surtout leur abondance en zone de forêt semi décidue. Ces 
résultats ont été observés par Orsot (2016) à Agboville lors des études ethnobotaniques. Par contre, N'guessan et al. (2009) ont montré que ce sont plutôt les Poaceae qui représentaient la famille la plus citée dans le traitement de l'hypertension artérielle.

Les phanérophytes, en particulier les microphanérophytes $(51,92 \%)$ ont constitué la plupart des types biologiques rencontrés dans l'étude. Ces observations sont similaires à celles de Lakouéténé et al. (2009) et Yapo (2017).

La phytogéographie a été dominée par les taxons communs à la région Guinéo-Congolaise et à la région Soudano-Zambézienne (GC-SZ). En effet, Yapo (2017) a montré, dans ses travaux, que les taxons communs à la région Guinéo-Congolaise et à la région Soudano-Zambézienne (GC-SZ) ont été majoritairement représentés à $46,67 \%$; ce que confirment nos résultats.

Les plantes ligneuses ont été les plus représentées du fait de leur abondance dans le département. Ces études viennent corroborer celles de Koulibaly et al. (2017). Ils ont montré que les plantes médicinales recensées étaient représentées à $67 \%$ par des plantes ligneuses.

\section{Caractéristiques ethnomédicinales}

La feuille (33\%) a été la partie de plantes la plus employée. Ces résultats sont en accord avec ceux de Zirihi (1991) avec 64,49\%, Ghourri et al. (2012) avec 73,28\%, Béné et al. (2017) avec 64\%, Kanga (2017) avec 43\% et Yapo (2017) avec 44\%. La fréquence d'utilisation élevée des feuilles pourrait être expliquée par l'aisance et la rapidité de la récolte (Bitsindou, 1986) mais également par le fait qu'elles sont le siège de la photosynthèse et du stockage des métabolites secondaires responsable des propriétés biologiques de la plante (Bigendako-Polygenis et Lejoly, 1990). De plus, le prélèvement intense des feuilles ne présente tout de même aucun danger pour la plante (Poffenberger et al., 1992). Selon cet auteur, le prélèvement de 50\% des feuilles d'un arbre n'affecte pas de manière significative la survie de celuici. Par contre l'écorçage laisse le plus souvent des cicatrices énormes par lesquelles ces plantes sont ultérieurement attaquées par les champignons, les oiseaux, les chenilles infestantes (Ouattara, 2006). Le déracinement, l'ébranchage, l'écorçage et l'abattage constituent des modes de prélèvement dangereux pour la plante.

Le mode de préparation couramment conseillé a été la décoction (69\%). Ces résultats ont été observés à des proportions moindres, par Adjanohoun et Aké-Assi (1979) avec 32,94\%, N'guessan et al. (2009) avec $42 \%$, Hachi et al. (2015) avec 34,57\%. La population locale croit au mode de décoction et le trouve adéquat pour réchauffer le corps et désinfecter la plante (Bwassiwe et al., 2014). Selon Mpondo et al. (2017) la décoction permet de recueillir le plus de principes actifs et atténue ou annule l'effet toxique de 
certaines recettes.

Les recettes proposées par les tradithérapeutes du département étaient majoritairement monospécifiques (98,05\%). Cette prépondérance est à l'avantage des patients. En effet, les associations de plantes mal assorties sont parfois dangereuses. En Afrique, environ 30\% des accidents mortels sont dus à l'usage des mixtures (El-Said et al., 1969). La prédominance des recettes monospécifiques confirment les résultats de Wangny (2013). L'auteur a révélé que 93,93\% des recettes étaient monospécifiques.

Dans le cadre du traitement de l'hypertension artérielle, la voie orale (93\%) a été le canal d'administration le plus employé. Cela pourrait s'expliquer d'une part, par l'aisance de ce moyen et d'autre part, par le fait que la maladie soit une affection liée à des organes profonds. Ainsi, pour atteindre ces organes, tout composé doit transiter par l'appareil digestif pour en faciliter son assimilation. Ces résultats ont été les mêmes que ceux de N'guessan et al. (2009) et de Dibong et al. (2011).

\section{Bases pharmacologiques et phytochimique des utilisations thérapeutiques traditionnelles}

La plupart des molécules dosées ont été présentes dans les espèces utilisées par les populations du département. L'effet hypotenseur proviendrait des groupes chimiques suivants : alcaloïdes, flavonoïdes, polyphénols, polyterpènes, saponosides et stérols comme l'ont suggéré les auteurs du tableau 7.

Tableau 7 : Activités pharmacologiques des groupes phytochimiques

\begin{tabular}{ll}
\hline $\begin{array}{l}\text { Groupes phytochimiques et activités } \\
\text { pharmacologiques }\end{array}$ & Auteurs \\
\hline $\begin{array}{l}\text { Flavonoïdes : Vasodilatateurs, inhibiteurs des } \\
\text { récepteurs adrénergiques }\end{array}$ & N'guessan et al. (2009) \\
\hline & Bruneton (1993) \\
\cline { 2 - 2 } Polyphénols : Effet hypotenseur & N'guessan et al. (2009) \\
\hline $\begin{array}{l}\text { Alcools (linatol, géraniol) et saponosides : } \\
\text { effet diurétique }\end{array}$ & Nacoulma (1996) \\
\hline $\begin{array}{l}\text { Alcaloïde de type réserpine : effet } \\
\text { hypotenseur }\end{array}$ & Kerharo et Adam (1974) \\
\hline Stérols et polyterpènes : action diurétique & Bouquet et Debray (1974) \\
\hline
\end{tabular}

\section{Conclusion}

L'enquête ethnobotanique menée a permis de dresser un catalogue de 52 espèces de plantes hypotensives. Ces taxons ont été répartis en 50 genres. Ils appartenaient à 30 familles botaniques avec une dominance des Fabaceae. 
Les ligneux ont été les plus abondants par rapport aux herbacées. Les taxons les plus représentés ont été ceux communs à la région Guinéo-Congolaise et à la région Soudano-Zambézienne (GC-SZ). La plupart des espèces hypotensives répertoriées ont été des microphanérophytes. Les feuilles ont été les organes végétaux les plus utilisés et la décoction a représenté le mode de préparation le plus employé. Les recettes médicamenteuses étaient généralement monospécifiques. L'administration des médicaments se faisait majoritairement par voie orale. Les effets thérapeutiques induits par les plantes, pour traiter l'hypertension artérielle ont été le fait de divers groupes chimiques : alcaloïdes, flavonoïdes, polyphénols, stérols et polyterpènes, qui constituent la base scientifique de l'utilisation thérapeutique traditionnelle des plantes utilisées. Ces propriétés thérapeutiques citées peuvent justifier l'intégration des plantes médicinales dans le programme d'aménagement forestier en vue de leur exploitation durable.

\section{Remerciements}

Nos remerciements vont aux chefs de village, aux habitants des villages, ainsi qu'aux herboristes et tradipraticiens du département de Divo pour leur disponibilité.

\section{References:}

1. Adjanohoun, E.J. \& Aké-Assi, L. (1979). Contribution au recensement des plantes médicinales de Côte d'Ivoire. Université d'Abidjan, Centre National de Floristique (C.N.F.), 358 p.

2. Anonyme (2003). Hypertension artérielle et ses complications. 2003 Institut de Cardiologie d'Abidjan, (Côte d'Ivoire). 183 pp.

3. Anonyme (2016). RGPH-2014 résultats globaux. Rapport du Secrétariat Technique $\quad$ Permanent, 22 p. http://www.ins.ci/n/templates/docss/RGPH2014D.pdf (Accès le 21/04/2019)

4. Aouinty, B., Oufara, S., Mellouki, F. \& Mahari, S. (2006). Evaluation préliminaire de l'activité larvicide des extraits aqueux des feuilles du ricin (Ricinus communis L.) et du bois de thuya (Tetraclinis articulata(Vahl) Mast.) sur les larves de quatre moustiques culicidés : Culex pipiens (Linné), Aedes caspius (Pallas), Culiseta longiareolata (Aitken) et Anopheles maculipennis (Meigen). Biotechnoogy Agronomy Society and Environment, 10 (2) : 67-71

5. APG IV (2016). An update of the Angiosperm Phylogeny Group classification for the orders and families of flowering plants. Botanical Journal of the Linnean Society, 181: 1-20. 
6. Béné, K., Camara, D., Fofié, N. B. Y., Kanga, Y., Yapi, A. B., Yapo, Y. C., Ambé, S. A. \& Zihiri, G. N.(2016). Etude ethnobotanique des plantes médicinales utilisées dans le département de Transua, District du Zanzan (Cote d'Ivoire). Journal of animal \& plant Sciences, 27 (2) : 4230-4250.

7. Békro, Y. A., Békro, J. A. M., Boua, B. B., Tra Bi, F. H. \& Éhilé, E. E.(2007). Etude ethnobotanique et screening phytochimique de Caesalpinia benthamiana (Baill.) Herend et Zarucchi (Caesalpiniaceae). Revue des Sciences Naturelles, 4 (2) : 217-225.

8. Bigendako-Polygenis, M. .J., \& Lejoly, J. (1990). La pharmacopée au Burundi. Pesticides et médicaments en santé animale, Presses Universitaires de Namur. pp 425-442.

9. Bitsindou, M. (1986). Enquête sur la phytothérapie traditionnelle à kindamba et odzala (Congo) et analyse de convergence d'usage médicinale en Afrique centrale. Mémoire de Document (inédit), Université Libre de Bruxelles, (Belgique), 482p.

10. Bouquet, A. \& Debray, M. (1974). Plantes médicinales de Côte-d'Ivoire. Imprimerie Louis-Jean, Paris, (France), pp 1-232

11. Bruneton, J. (1993). Pharmacognosie, Phytochimie, plantes médicinales, 2nde Ed, Technique et Documentation Lavoisier, Paris, $915 \mathrm{p}$

12. Bwassiwe, H., Metowogo, K., Aklesso, P., Mouzou, R., Tossou, R., Ahounou, J., Eklu- Gadegbekou, K., Dansou, P. \& Aklikokou, K. (2014). Enquête ethnobotanique sur les plantes utilisées dans le traitement traditionnel des contusions musculaires au Togo. Revue. Ivoirienne. Sciences et Technologie, 24: 112 - 130.

13. Cunningham, A. B. (2002). Applied ethnobotany: People, wild plant use and conservation. Earth scan publications, $300 \mathrm{p}$

14. 14- Diallo, D., Guissou, I. P., Haïdara, M., Tall, C., Kasilo, O. M. J. (2010). Recherche sur la médecine traditionnelle africaine : hypertension. African haelth monitor, 13

15. Diatta, C. D., Gueye, M. \& Akpo, L. E. (2013). Les plantes médicinales utilisées contre les dermatoses dans la pharmacopée Baïnounk de Djibonker, région de Ziguinchor (Sénégal). Journal of Applied Biosciences, 70 : 5599-5607.

16. Dibong, S. D., Mpondo, M. E., Ngoye, A., Kwin, M. F. \& Betti, J. L. (2011). Ethnobotanique et phytomédecine des plantes médicinales de Douala, Cameroun. Journal of Applied Biosciences, 37 : 2496-2507. 
17. El-Said, F., Sofowora, E., Malcolm, A. \& Hoffer, A. (1969). An investigation into the efficacy of Ocimum gratissimum L. (Lamiaceae) as used in Nigeria native medicine, Planta Medica, 97: 195-200.

18. Gentilini, M. (1993). Médecine tropicale. Edition Flammarion, Paris, $928 \mathrm{p}$.

19. Ghourri, M., Zidane, L., Houda, E. Y., Rochdi, A., Fadli, M. \& Douira, A. (2012). Etude floristique et ethnobotanique des plantes médicinales de la ville d'El Ouatia (Maroc Saharien). Journal of Forestry Faculty, $12(2): 218-235$

20. Hachi, M., Hachi, T, Belahbib, N., Dahmani, J. \& Zidane, L. (2015).Contribution à l'étude floristique et ethnobotanique de la flore médecinale utilisée au niveau de la ville de Khenifra (Maroc). International Journal of Innovation and Applied Studies 11 (3) : 754770

21. Hegnauer, R. (1973). Chemotaxonomie der Pflanzen, Bikhäuser Verlag, Basel, Suttgart, 797 p.

22. Kanga, Y. (2017). Enquête ethnobotanique sur les plantes médicinales de la Région du Haut Sassandra (Côte d'Ivoire) et évaluation des activités pharmacologiques de deux taxons sollicités dans le traitement des infections cutanées. Thèse de Doctorat, Université Felix Houphouët-Boigny de Cocody, Abidjan, (Côte d'Ivoire), 200 p.

23. Kearney P. M, Whelton M., Reynold K., Muntner P., Whelton P. K. \& He J. (2005). « Global Burden of hypertension: analysis of world wide data ». The Lancet, 365 (9455) : 217-230.

24. Koffi, A. (2007). Prise en charge des patients adultes atteints d'hypertension artérielle à l'institut de Cardiologie d' Abidjan (Côte d'Ivoire) : Rapport/ICA, 6 p.

25. Kerharo, J. \& Adam, J. G. (1974). La pharmacopée sénégalaise traditionnelle. Plantes médicinales et toxiques. Édition Vigot frères, Paris, pp. 100-950

26. Koulibaly, A., Monian, M., Ackah, J. A. A. B., Koné, M. W. \& Traore, K. (2017). Étude ethnobotanique des plantes médicinales : cas des affections les plus fréquentes d'une région agricole Daloa (Centre Ouest, Côte d'Ivoire). Journal of Animal \&Plant Sciences, 31 (2) : 5021-5032.

27. Lakouéténé, D.P.B., Ndolngar, G., Berké, B., Moyen, J-M., Kosh Komba, E., Zinga, I., Silla, S., Millogo-Rasolodimby, J., Vincendeau, P., Syssa-Magalé, J-L., Nacoulma-Ouedraogo, O. G., Laganier, R., Badoc, A. \& Chèze,C. (2009). Enquête ethnobotanique des plantes 
utilisées dans le traitement du paludisme à Bangui. Bulletin de la. Société de Pharmacie de Bordeaux, 148 : 123-138.

28. Mpondo, M. E., Ngene, J. P., Som, M. L., Etame, L. G., Boumsong, N. P.C., Yinyang, J. \& Dibong, S. D. (2017). Connaissances et usages traditionnels des plantes médicinales du département du haut Nyong. Journal of applied Biosciences 113 : 11229-11245.

29. Nacoulma-Ouédraogo, O. (1996). Plantes médicinales et pratiques médicales traditionnelles au Burkina Faso : cas du Plateau central. Thèse de doctorat ès sciences naturelles, faculté de science et technique, université de Ouagadougou, (Burkina-Faso), pp. 25-605

30. Ngoule C., Ngene J., Kidick P., Ndjib R., Dibong S. \& Mpondo, M. E. (2015). Inventaire et caractérisation floristiques des plantes médicinales à huiles essentielles des marchés de Douala Est (Cameroun). International. Journal of Biological and. Chemical Sciences, 9 (2) : 847-889.

31. N'Guessan, K., Kadja, B., Zirihi, G., Traoré, D. \& Aké-Assi, L. (2009). Screening phytochimique de quelques plantes médicinales ivoiriennes. Sciences \& Nature, $6: 1$ - 15 .

32. N’Guessan, K., Tiébre, M. S., Aké-Assi, E. \& Zirihi, G. N. (2009). Ethnobotanical study of plants used to treat arterial hypertension, in traditional medicine, by Abbey and Krobou populations of Agboville (Côte d'Ivoire). European Journal of Scientific Research, 35 (1) : 85 98.

33. N'guessan, K., Soro, D. \& Amon, A. D. E. (2011). Plantes utilisées en médecine traditionnelle dans le traitement des maladies cardiovasculaires, en pays Abbey et Krobou, dans le Sud de la Côte-d'Ivoire. Article de synthèse EthnopharmacologiePhytothérapie, Springer-Verlag France, 9 : 199-208.

34. Orsot, B. (2016). Etude ethnobotanique des plantes médicinales utilisées dans le traitement des maladies de la peau par les Abbey du Département d'Agboville (Côte d' Ivoire) et évaluation de l'activité antifongique des extraits de quatre plantes sur Sclerotium rolfsii, un phytopathogène. Thèse de Doctorat de l'Université Felix HouphouëtBoigny, Abidjan, (Côte d'Ivoire), 200p.

35. Ouattara, D. (2006). Contribution à l'inventaire des plantes médicinales significatives utilisées dans la région de Divo (sud forestier de la Côte-d'Ivoire) et à la diagnose du poivrier de Guinée : Xylopia aethiopica (Dunal) A. Rich. (Annonaceae). Thèse de doctorat de l'université de Cocody-Abidjan (Côte-d'Ivoire), 184p 
36. Poffenberger, M., Gean, B. M., Khare, A. \& Campebell, J. (1992). Field method manuel, Volume II. Community forest economy and use patterns: participary rural apprasail (P.R.A.) Methods in south Gujarat, India. Society for promotion of Wasteland developement, New dehli, pp 16-57.

37. Ronchetti, F. \& Russo, G. (1971). A new alkaloid from Rauvolfia vomitoria. Phytochemistry, $10: 1385-1388$.

38. Wagner, H. (1983). Drogen analyse. Dünschicht chromatographische analyse von Arzneidrogen. Springer Verlag Heidelberg New York, $522 \mathrm{p}$.

39. Wangny, A. A. S. (2013). Etude ethnobotanique des plantes médicinales hypotensives vendues sur les marchés de la commune de Marcory, dans le District d'Abidjan (Côte d'Ivoire). Mémoire Master II de botanique, Université Félix Houphouët- Boigny, (Côte d'Ivoire), $50 \mathrm{p}$.

40. Yapo, Y. C. V. (2017). Plantes médicinales antimicrobiennes utilisées dans la région des grands ponts (Cote d'Ivoire) : Etude ethnobotanique et évaluation des activités antibactériennes, antifongique et toxique des taxons les plus sollicités. Thèse de Doctorat National, Université Felix Houphouët-Boigny, (Côte d'Ivoire), 178p.

41. Zirihi, G. N. (1991). Contribution au recensement, à l'identification et à la connaissance de quelques espèces végétales utilisées dans la médecine traditionnelle et la pharmacopée chez les Bété du département d'Issia, Côte-d'Ivoire. Thèse de doctorat de troisième cycle, Université d'Abidjan, (Côte d'Ivoire), 150p 


\section{Annexe}

Images de quelques plantes médicinales (Photo WANGNY, 2016-2017)

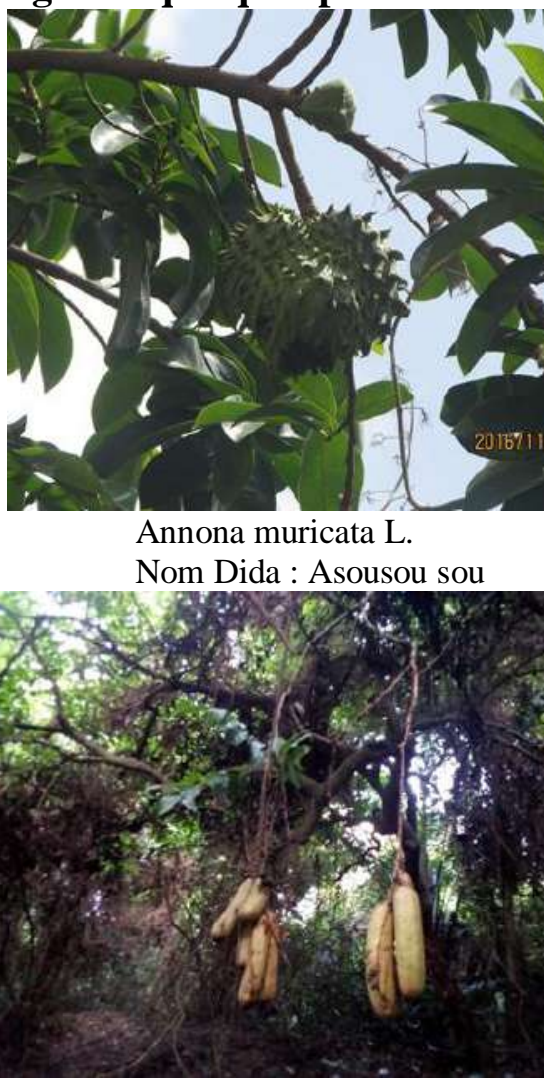

Kigelia africana (Lam.) Benth.

Nom Dida : Gnintissou

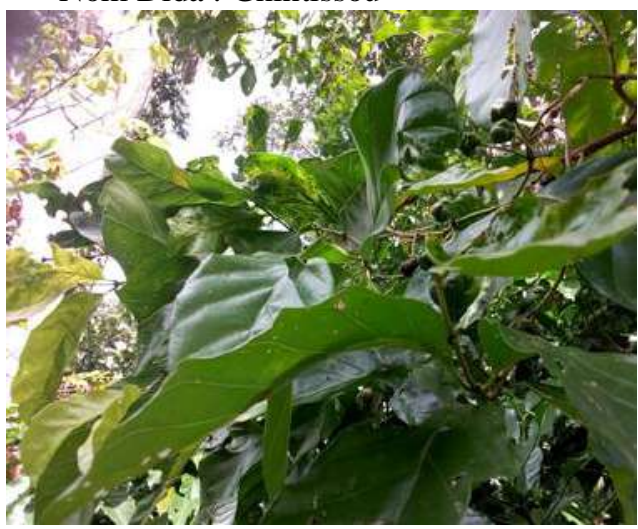

Morinda lucida Benth.

Nom Dida : Monessignakpè

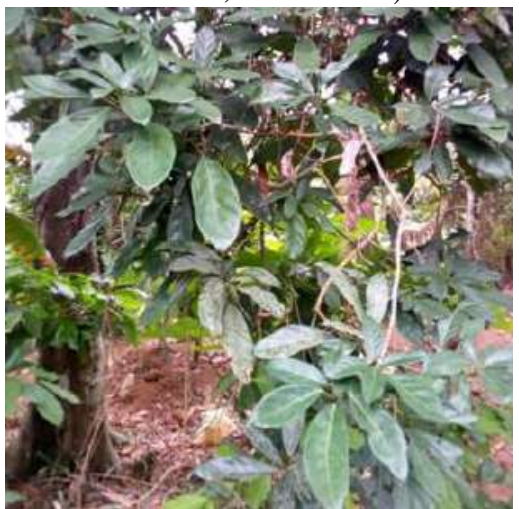

Cola nitida (Vent) Schott. Endl Nom Dida : Gouhèlè sou

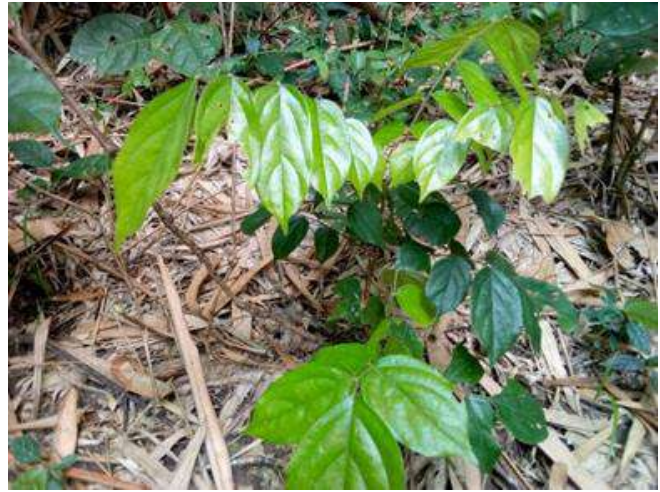

Manotes longiflora Baker

Nom Dida : Zagonoguié

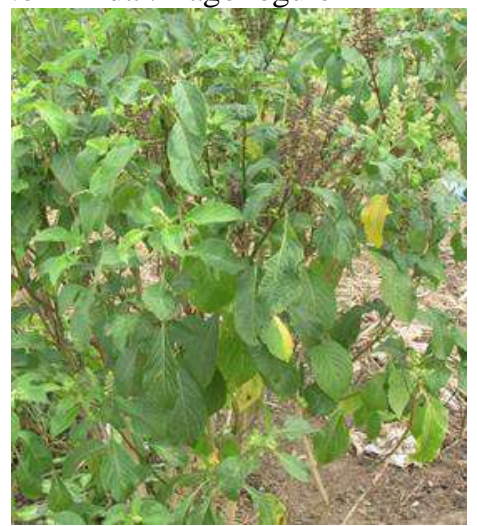

Ocimum gratissimum L.

Nom Dida : Mocolou 


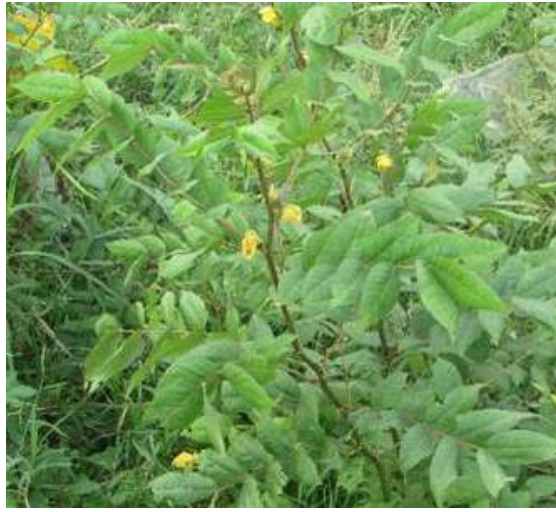

Senna occidentalis L.

Nom Dida : Gbossoudjètè

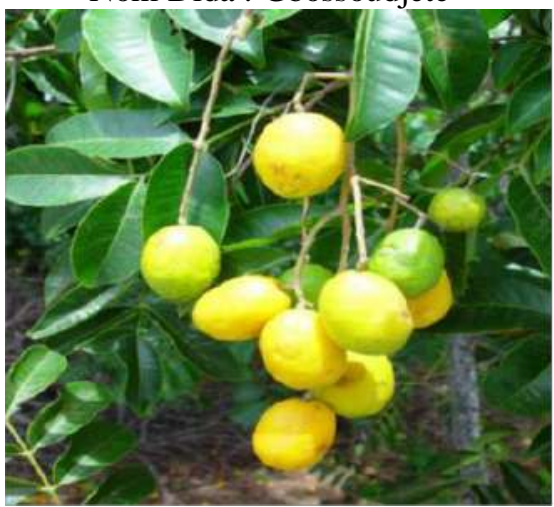

Spondias mombin L

Nom Dida : Totrosou

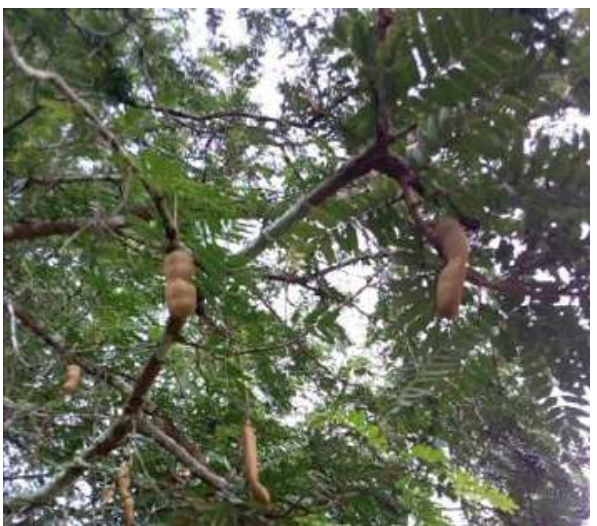

Tamarindus indica Linn.

Nom Malinké : Tomiyiri

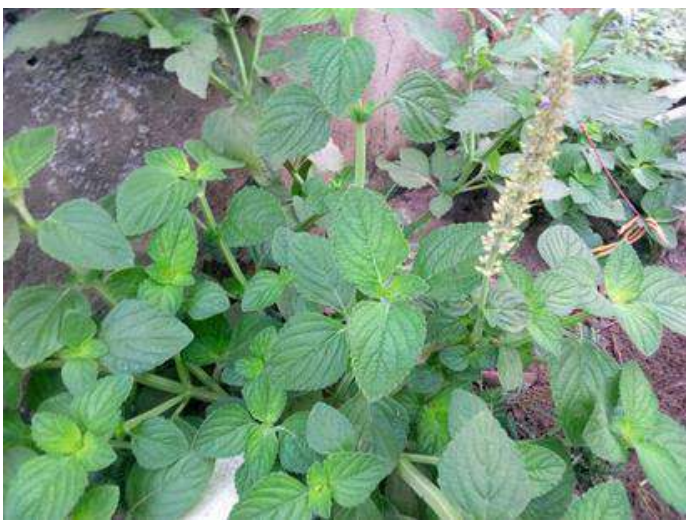

Solenostemon monostachyus (P.Beauv.)

Briq. Subsp Nom Dida : Boussagnon

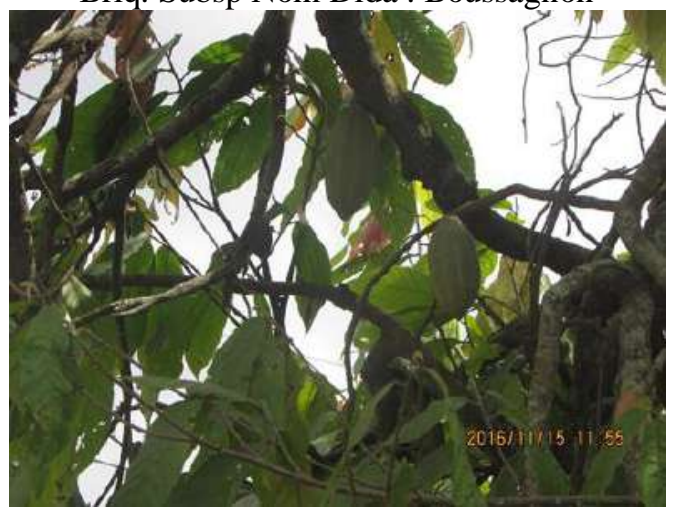

Theobroma cacao L.

Nom vernaculaire : Cacao

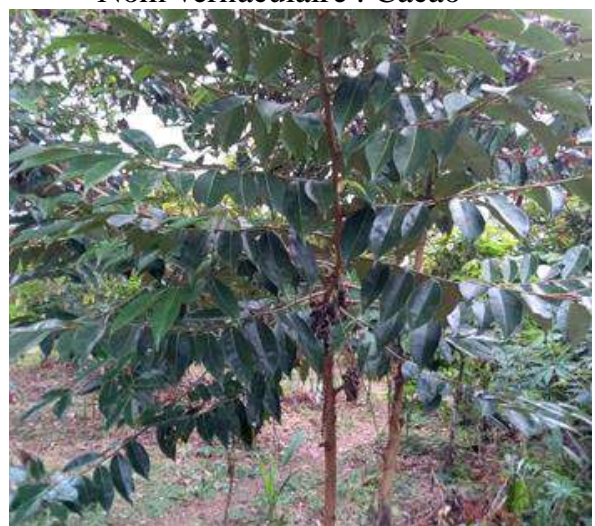

Xylopia aethiopca (Dunal.) A. Roch.

Nom Dida : Loulou 